\title{
Suivi de la coagulation-présure du lait à l'aide d'un nouveau capteur réfractométrique
}

\author{
J. KOROLCZUK*, J.-L. MAUBOIS* et J. LOHEAC** \\ * I.N.R.A., Laboratoire de Recherches de Technologie laitière \\ 65, rue de Saint-Brieuc, 35042 Rennes cedex, France \\ ** Société SOPELEM, 125, bd Davout, 75020 Paris, France
}

\section{Résumé}

Un réfractomètre prototype récemment développé par la société Sopelem était couplé avec un viscosimètre « Rhéomat 30 » (Contraves, Suisse) à cylindres coaxiaux dans le but de comparer les signaux issus de ces capteurs lors de la coagulation du lait par la présure. L'évolution de ces signaux au cours de la gélification était suivie à l'aide d'une interface et d'un microordinateur.

L'excellence des corrélations existant entre les deux familles de courbes traduisant respectivement l'évolution de la viscosité et celle du signal réfractométrique, ainsi que la similarité des résultats obtenus en leur appliquant l'équation de ScotT-Blair et BURNETT (1963), amenait à conclure que les deux capteurs mesuraient bien le changement d'état physique du lait sous l'effet de la présure et le raffermissement du gel. De ce fait, sous réserves des multiples vérifications à réaliser pour connaître l'effet des différents paramètres intervenant dans la coagulation du lait, le détecteur réfractométrique testé pourrait devenir de par son principe optique le capteur idéal pour l'automatisation des cuves de fromagerie.

L'effet de l'abaissement du pH d'emprésurage jusqu'à pH 5,6 était étudié et discuté. La remontée de la valeur maximale du signal réfractométrique en dessus de $\mathrm{pH}$ 6,0 accompagnant une exsudation de lactosérum amenait à émettre l'hypothèse que l'équipement réfractométrique testé pourrait également servir de capteur de suivi de la synérèse des caillés de fromagerie.

Mots clés : Réfractomètre - Viscosimètre - Capteurs de fromagerie - Automatisation Coagulation présure - $\mathrm{pH}$ - Synérèse.

\section{Summary}

Rennet coagulation of milk followed by a new refractometer detector

A new prototype refractometer recently built by the French Society Sopelem was connected to a coaxial cylinder viscosimeter "Rheomat 30" (Contraves - Switzerland) in order to compare output signals from both sensors during rennet coagulation of milk. Evolution of both output signals was followed through an interface coupled with a personal microcomputer. 
The high level of correlation existing between both curves families respectively describing viscosity evolution and the growth of refractometric output signal, the similarity of data obtained by treating these curves with the mathematical model proposed by ScOoT-BLAIR and BURNETT (1963) led to conclude that both sensors were well measuring changes in physical state of milk after rennet action and gel hardenings. Consequently, the tested refractometric unit could be the ideal sensor for cheese vats automatization because of its optical principle. But, before an industrial spreading, numerous studies are required to know effects of the multiple parameters acting on rennet coagulation.

Effect of lowering renneting $\mathrm{pH}$ until $\mathrm{pH} 5,6$ was tested and discussed. The increase of maximal value of refractometric output signal under $\mathrm{pH} 6,0$ joined with whey expelling led to the hypothesis that the tested refractometric unit could be also a sensor for following cheese curd syneresis.

Key words : Refractometer - Viscosimeter - Cheesemaking sensors - Rennet - Coagulation - pH - Syneresis - Automatization.

\section{Introduction}

De nombreux capteurs ont été proposés pour mesurer l'évolution des caractéristiques rhéologiques du lait au cours de sa coagulation sous l'effet de la présure. Leurs principes et leurs domaines possibles d'application ont été revus récemment (KoROLCZUK et al., 1986).

La mesure de l'indice de réfraction est utilisée depuis de nombreuses années pour suivre la concentration par évaporation du lait sucré ou non (RICE et Miscall, 1926, Goulden, 1963). Elle est utilisée depuis la fin des années 70 comme base des capteurs de régulation de la concentration du lait ou de ses dérivés par ultrafiltration sur membrane (Boudier et al., 1977 ; MAHAUT, 1981).

L'indice de réfraction d'une solution complexe comme le lait est une grandeur physique globale dans laquelle s'additionnent les effets sur la réfraction de la lumière des composants protéiques (caséines et protéines solubles) et des solutés de la phase aqueuse (lactose et sels minéraux). Ces composants interviennent par leur concentration mais aussi par leur taille (RoBERTSON, 1918). Dès 1929, DUMANSKII et PuchKonski ont proposé la relation suivante entre la valeur de l'indice de réfraction des solutions de caséine et le diamètre micellaire :

$$
\mathrm{n}=\mathrm{C}_{1}\left(\mathrm{n}_{2}-\mathrm{n}_{1}\right) / \mathrm{d}_{2}+\mathrm{n}_{1}
$$

où $\mathrm{n}, \mathrm{n}_{1}$ et $\mathrm{n}_{2}$ sont respectivement les indices de réfraction de la solution, du solvant et des micelles de caséine, $\mathrm{d}_{2}$ le diamètre des micelles dispersées et $\mathrm{C}_{1}$ la concentration en caséine. Il résulte de cette relation que tous les paramètres de milieu diminuant le volume en solution des micelles de caséine (acidification, traitement thermique, force ionique, etc.) accroîtront à concentration constante l'indice de réfraction de la solution et vice versa.

Sur un autre plan, il est bien connu que la turbidité d'une solution macromoléculaire est directement proportionnelle à la taille des molécules en solution et qu'en conséquence toute agrégation micellaire se traduit par un accroissement de la diffusion lumineuse. Or l'intensité de cette diffusion est elle-même étroitement dépendante d'une variation de l'indice de réfraction (STACEY, 1961). 
Ces considérations nous ont conduits à penser que la coagulation enzymatique du lait sous l'action de la présure, qui induit un réarrangement spatial des micelles de caséine avec formation d'un réseau tridimensionnel, pouvait également entraîner un changement de l'indice de réfraction au cours de la formation du gel.

Pour ce faire, nous avons utilisé un réfractomètre prototype développé récemment grâce au concours financier de la Direction des Industries Agricoles et Alimentaires (contrat $\mathrm{n}^{\circ} 651$ 154) par la société Sopelem (France) et qui a été associé avec un viscosimètre à cylindres coaxiaux dans le but de comparer l'évolution du signal de sortie du réfractomètre avec celle de la viscosité apparente d'échantillons de lait emprésurés en cours de coagulation.

\section{Matériel et méthodes}

Lait utilisé : une poudre de lait écrémé commerciale, de qualité * Low heat », était reconstituée à $10 \%$ de matière sèche $(100 \mathrm{~g}$ dans $900 \mathrm{~g}$ d'eau distillée) - 0,02\% (p/v) d'azoture de sodium (Merck, R.F.A.) étaient ajoutés pour prévenir tout développement bactérien. Le $\mathrm{pH}$ était ajusté à la valeur souhaitée (entre 6,6 et 5,6 ), à $20^{\circ} \mathrm{C}$ à l'aide de $\mathrm{NaOH} 0,5 \mathrm{~N}$ ou d'HCl $0,5 \mathrm{~N}$. Avant l'addition de présure (poudre commerciale Hansen reconstituée à $1 \mathrm{~g}$ dans $100 \mathrm{ml}$ de tampon acétate pH 5,5 additionné de 0,02\% d'azoture de $\mathrm{Na}$ ), le lait reconstitué était maintenu $1 \mathrm{~h}$ au bain-marie à la température souhaitée.

Réfractomètre utilisé : l'équipement ayant servi aux expérimentations est un prototype spécialement développé par la société Sopelem (France) pour l'insertion en ligne comme capteur de régulation des systèmes d'ultrafiltration. Ce réfractomètre de marque Recor était pour les besoins de l'expérimentation fixé au fond d'une cuve à double paroi servant de cylindre externe thermostaté à $35^{\circ} \pm 0,1^{\circ} \mathrm{C}$ au viscosimètre «Rhéomat $30 »$ (Contraves, Suisse) (fig. 1). Un seul gradient de vitesse était utilisé pour la mesure de la viscosité cinématique : $2347 \mathrm{~s}^{-1}$.

Le réfractomètre Recor comporte trois cellules photo-électriques mesurant la luminosité dans les zones illuminée, transitoire et sombre (fig. 2). Le potentiel (R) de sortie exprimé en volts résulte de la transformation des trois mesures de la luminosité selon la relation suivante :

$$
\mathrm{R}=\frac{\mathrm{T}-\mathrm{S}}{\mathrm{I}-\mathrm{S}}
$$

où $\mathrm{R}$ est le signal de sortie du réfractomètre ;

$\mathrm{I}$, $\mathrm{T}$ et $\mathrm{S}$ sont les signaux émanant respectivement des trois photodétecteurs placés en zones illuminée, transitoire et sombre.

Le signal de sortie (R) est électroniquement linéarisé. L'équation découlant de cette linéarisation dans la gamme 3,5 à 10 volts (soit pour des solutions de concentration croissante en saccharose : 7,5 à $22,3 \mathrm{~g} \mathrm{p} .100 \mathrm{~g}$ ) est égale à : $\mathrm{Ir}=1,3223+0,0035195 . \mathrm{R}$ avec $\mathrm{r}=$ coefficient de corrélation égal à 0,9998 ou Ir est l'indice de réfraction exprimé en unités réfractométriques. 


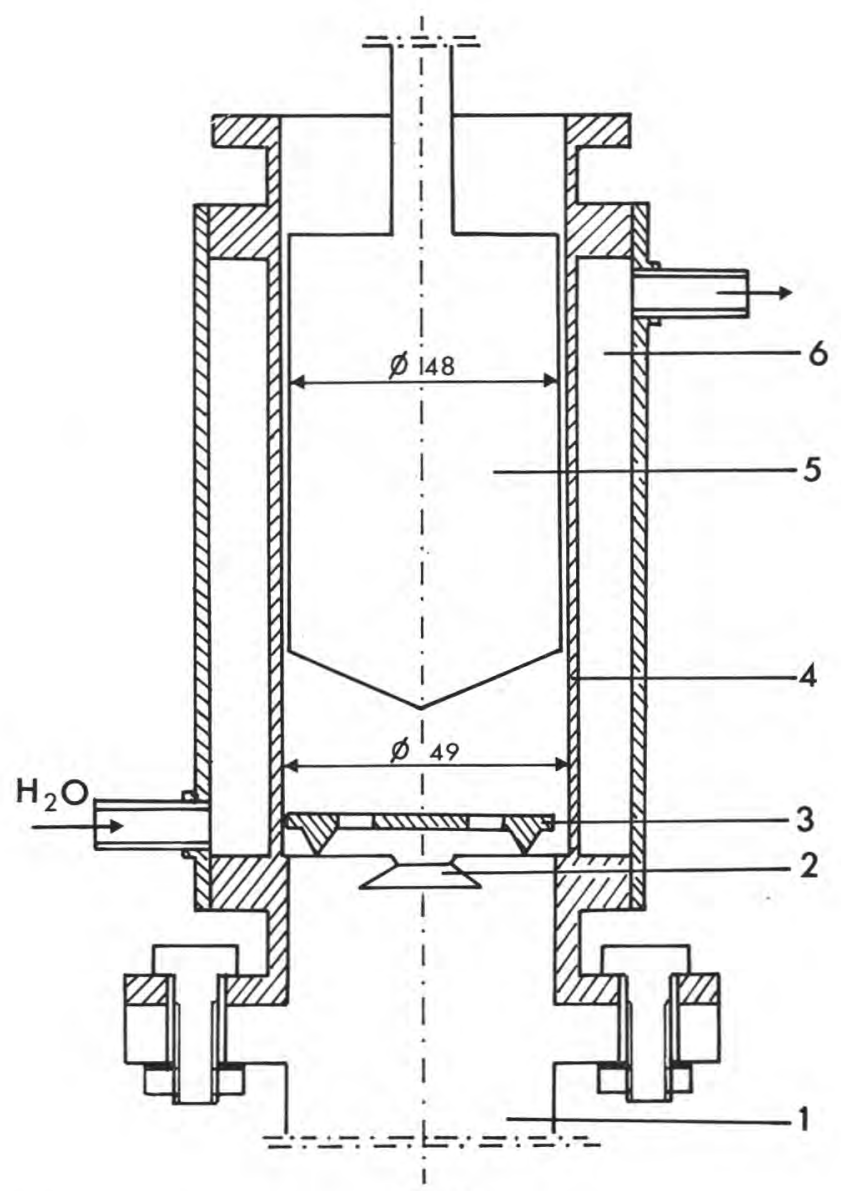

Fig. 1

Schéma du couplage du réfractomètre Recor avec le viscosimètre Rhéomat $30 ; 1$ : Réfractomètre - 2: Prisme du réfractomètre - 3: Disque ajouré d'isolement de la zone de mesure réfractométrique - 4: Cylindre externe du viscosimètre - 5: Cylindre interne du viscosimètre - 6 : Jaquette de thermostatation.

Schema of the connection of Recor refractometer with Rheomat 30 viscosimeter ; 1: Refractometer - 2: Refractometer prism - 3:Ring gasket - 4: External cylinder of the viscosimeter - 5: Internal cylinder of the viscosimeter - $6:$ Water jacket.

Nous avons constaté que la résolution du réfractomètre était meilleure que $0,1 \mathrm{mVolts}$ soit $3,5 \times 10^{-7}$ unités réfractométriques.

Acquisition et traitement des signaux : le viscosimètre et le réfractomètre étaient reliés à un convertisseur analogique/digital (type CAN 1612 M1 - M.I.D., France) lui-même connecté à un micro-ordinateur Apple II plus, U.S.A. L'acquisition des données était réalisée à l'aide du logiciel décrit par KorolczuK et al. (1985). La moyenne arithmétique de 50 mesures des deux signaux provenant des équipements était enregistrée toutes les $5 \mathrm{~s}$ simultanément au temps délivré par l'horloge interne du micro-ordinateur (Glanmire Electronics, Irlande). De 900 


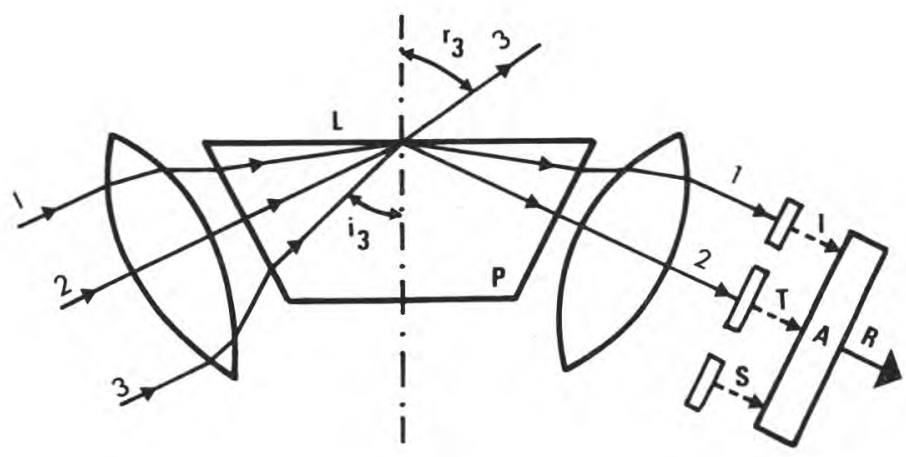

Fig. 2

Principe de fonctionnement du réfractomètre Recor : $L$ - Liquide à contrôler ; $P$ Prisme ; I, T, S - Cellules photoélectriques placées respectivement dans les zones : illuminée, transitoire et sombre; $A$ - Amplificateur ; $R$-Signal de sortie ; 1, 2, 3 - Rayons de la lumière monochrome; $i_{3}$ - Angle d'incidence du rayon $3 ; r_{3}$ - Angle de réfraction du rayon 3 .

Functionning principle of the refractometer Recor : L - Analyzed liquid ; P - Prism ; $I, T, S$ - Photodetectors placed respectively in the illuminated, transitory and dark zone; A - Amplifier ; $R$ - Output signal ; $1,2,3$ - Rays of monochromatic light ; $i_{3}$ - Angle of incidence of the ray $3 ; r_{3}$ - Angle of refraction of the ray 3 .

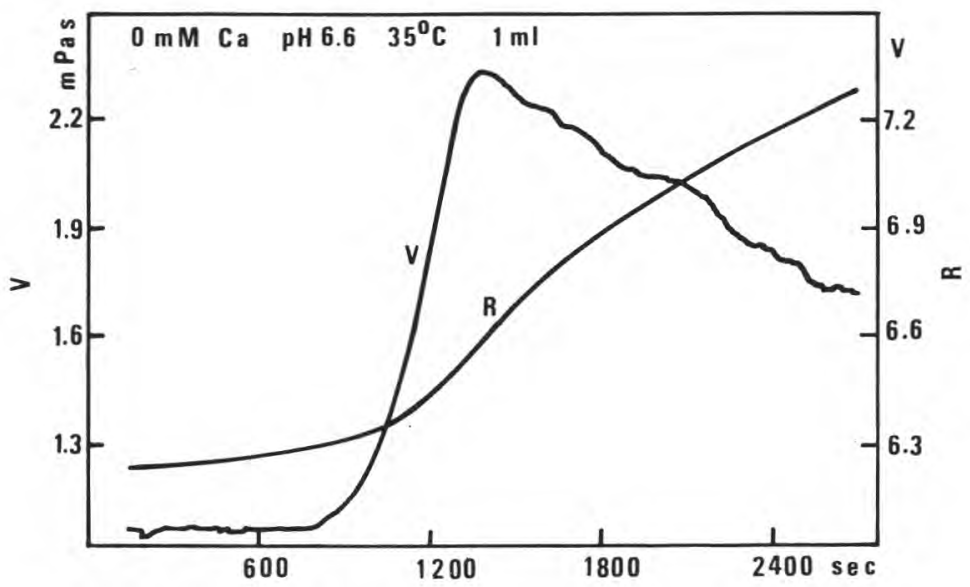

Fig. 3

Evolutions de la viscosité apparente $(V)$ et du signal de sortie du réfractomètre (R) au cours de la coagulation du lait par la présure. Conditions expérimentales : présure en poudre $/ N \times 6,38: 1 / 300$; température $35^{\circ} \mathrm{C} ; \mathrm{pH}: 6,6$; pas de calcium ajouté ; gradient de vitesse du viscosimètre : $2347 s^{-1}$.

Apparent viscosity $(V)$ and refractometric output signal $(R)$ evolutions during rennet coagulation of milk. Experimental conditions : rennet powder $/ N \times 6,38$ of milk $1 / 300$; température : $35^{\circ} \mathrm{C} ; \mathrm{pH} 6,6 ;$ no added calcium rate of strain : $2347 \mathrm{~s}^{-1}$. 
à 2000 triplets de mesures étaient ainsi enregistrés sur disque souple de 2 mégaoctets (Micro Expansion, France) pour chaque expérimentation.

A partir de résultats expérimentaux, le temps de gélification et les valeurs maximales du signal réfractométrique étaient calculés à l'aide du modèle mathématique de Scott-Blair et Burnett (1963) selon la méhode décrite précédemment (KorolcZuK et al., 1986).

\section{Résultats}

La figure 3 est un exemple de l'évolution concomitante en fonction du temps du signal de sortie du réfractomètre ( $R$ ) et de la viscosité apparente (V) durant la coagulation par la présure du lait. Il y a une bonne similitude des accroissements des signaux $\mathbf{R}$ et $\mathrm{V}$ durant la phase initiale de gélification du lait. Ensuite, la destruction du gel produite par la rotation du cylindre interne du viscosimètre se traduit évidemment par une chute de la viscosité apparente. Au contraire, le gel au contact de la fenêtre prismatique du réfractomètre n'étant pas mécaniquement brisé, continue à se raffermir et donc le signal de sortie émanant de cet équipement à croître. La forme de la courbe traduisant l'évolution en fonction du temps de $(\mathrm{R})$ est très similaire à celles représentatives de l'évolution des propriétés visco-élastiques des coagulum présure de lait obtenues avec des rhéomètres non destructeurs de gel (Culioli et Sherman, 1978 ; Douillard, 1973).

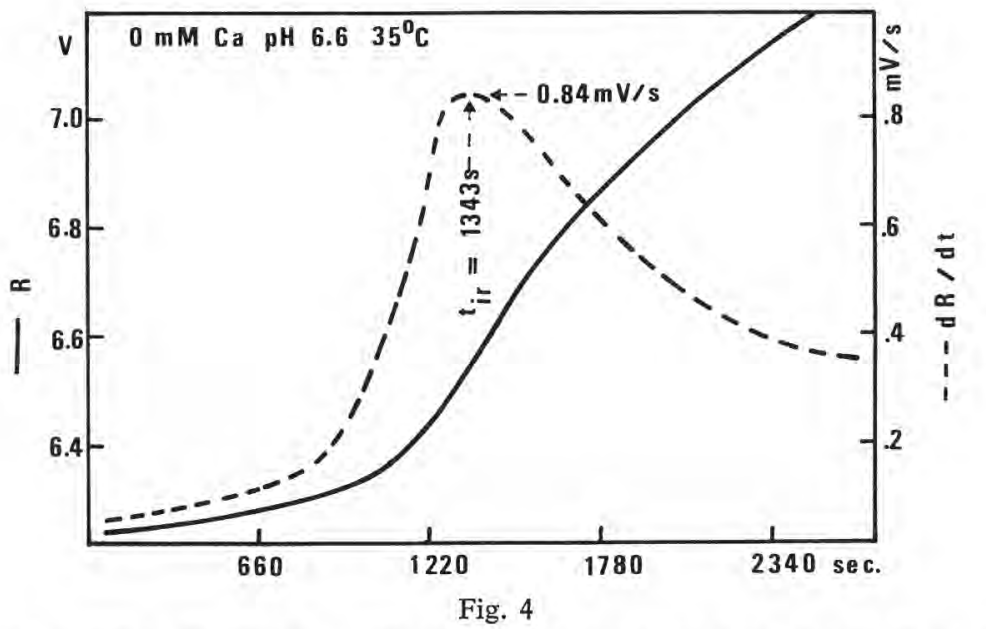

Evolutions $d u$ signal de sortie $d u$ réfractomètre (ligne en trait plein) et de la courbe dérivée en fonction du temps (ligne en pointillé) au cours de la coagulation du lait par la présure. Les conditions expérimentales sont les mêmes que celles de la figure 3.

Evolutions of output signal of the refractometer (continuous line) and of its derivative curve versus time (dotted line) during rennet coagulation of milk. Experimental conditions were the same as described in figure 3. 


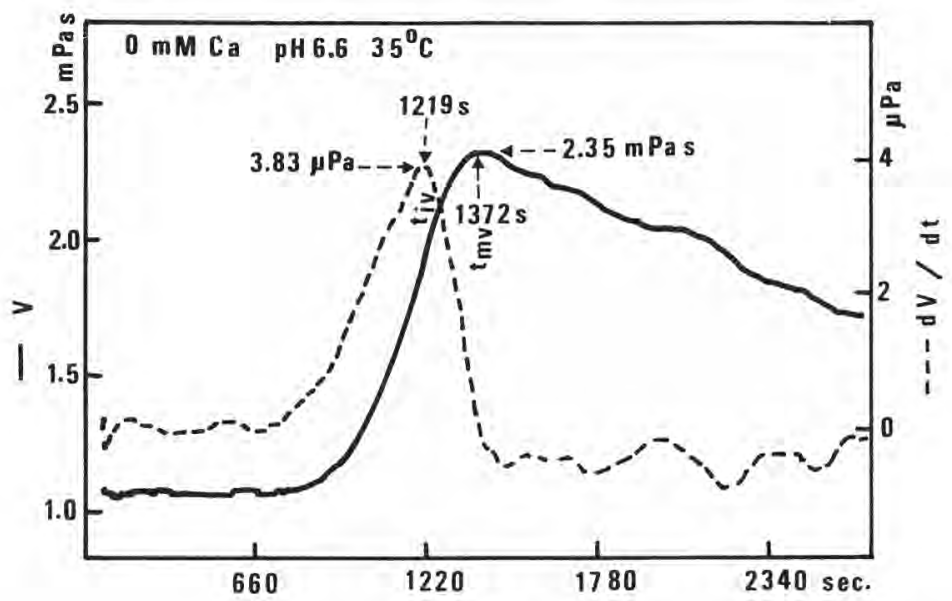

Fig. 5

Evolutions de la viscosité apparente (ligne en trait plein) et de la courbe dérivée en fonction du temps (ligne en pointillé) au cours de la coagulation du lait par la présure. Les conditions expérimentales sont les mêmes que celles de la figure 3.

Evolutions of the apparent viscosity (continuous line) and of its derivative versus time (dotted line) during rennet coagulation of milk. Experimental conditions were the same as described in figure 3.

Pour comparer les signaux émanant des deux équipements (réfractomètre et viscosimètre), les courbes des dérivées en fonction du temps $\mathrm{dR} / \mathrm{dt}$ et $\mathrm{dV} / \mathrm{dt}$ étaient calculées. Elles sont représentées respectivement dans les figures 4 et 5 . La courbe $\mathrm{dR} / \mathrm{dt}$ est très voisine de celle représentant $\mathrm{V}=\mathrm{f}(\mathrm{t})$ et son maximum correspondant au point d'inflexion de $R=f(t)$, obtenu pour $t=1343 \mathrm{~s}$, ne diffère guère de celui observé pour la viscosité $(t=1372 \mathrm{~s})$.

Une corrélation linéaire (fig. 6) était observée entre les temps de gélification calculés pour les deux familles de courbes $V=f(t)$ et $R=f(t)$, obtenues lors d'expérimentations où le rapport pondéral $\mathrm{N} \times 6,38$ /présure en poudre variait entre $30 / 1$ et $300 / 1$. Toutefois, les temps de gélification estimés à partir des courbes $\mathrm{R}=\mathrm{f}(\mathrm{t})$ étaient de $15 \%$ supérieurs à ceux calculés à partir des courbes viscosimétriques. Une excellente corrélation linéaire $(r>0,999)$ était également mise en évidence entre les temps de gélification obtenus à partir des courbes $V=f(t)$ et les temps $\left(t_{i r}\right)$ correspondant aux maxima des courbes $d R / d t$, c'est-àdire aux points d'inflexion des courbes représentant les signaux de sortie du capteur réfractométrique (fig. 6). Il en était de même entre les temps correspondant aux points d'inflexion des courbes $R=f(t)$ et les temps correspondant d'une part à la gélification ( $\left.\mathrm{t}_{\mathrm{gv}}\right)$, d'autre part aux maxima $\left(\mathrm{t}_{\mathrm{mv}}\right)$ des courbes viscosimétriques. Les relations existantes sont :

$$
\begin{gathered}
\mathrm{t}_{\mathrm{ir}}=13,7+1,19 \times \mathrm{t}_{\mathrm{gr}} ; \mathrm{r}=0,999 \\
\mathrm{t}_{\mathrm{mV}}=21,7+1,00 \times \mathrm{t}_{\mathrm{ir}} \text { avec } \mathrm{r}=0,9998
\end{gathered}
$$

De même, une corrélation linéaire existait entre les temps correspondant aux points d'inflexion des courbes réfractométriques et les temps de gélification estimés à partir des courbes $\mathrm{V}=\mathrm{f}(\mathrm{t})$ :

$$
\mathrm{t}_{\mathrm{ir}}=19,7+1,29 \times \mathrm{t}_{\mathrm{gv}} \text { avec } \mathrm{r}=0,999 \text { (fig. 6) }
$$




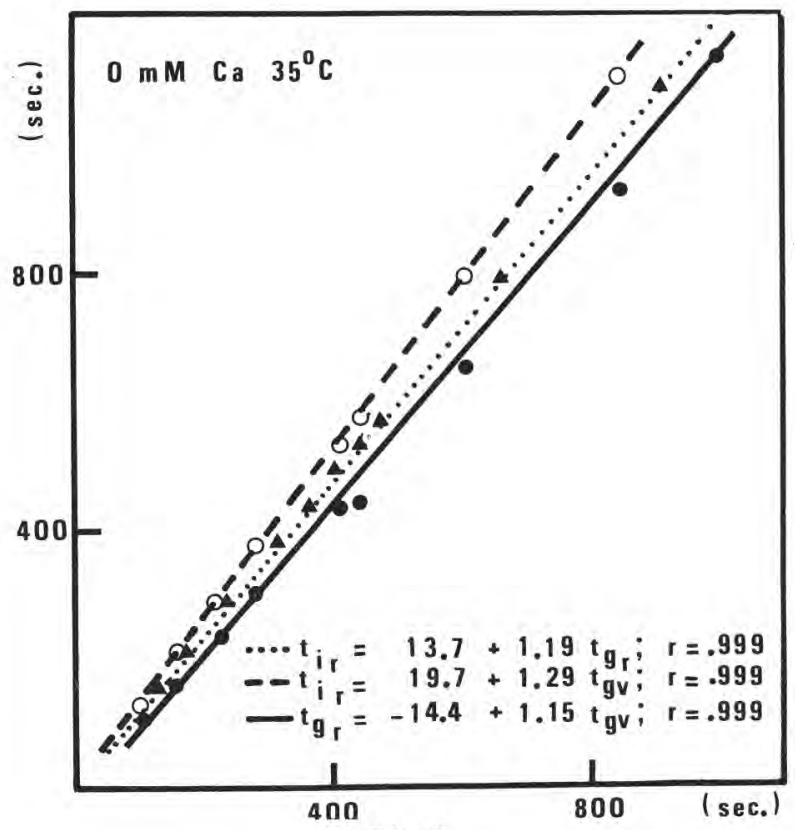

Fig. 6

Corrélations entre: a) Les temps aux points d'inflexion $\left(t_{i r}\right)$ des courbes réfractométriques et les temps de gélification $\left(t_{\text {qv }}\right)$ calculés à partir des courbes viscosimétriques (--) ; b) Entre $\left(t_{i r}\right)$ et les temps de gélification $\left(t_{g r}\right)$ calculés à partir des courbes réfractométrique $(\ldots) ; e)$ Entre $\left(t_{g r}\right)$ et $\left(t_{g v}\right)(-)$. Les conditions expérimentales étaient les mêmes que celles de la figure 2 à l'exception du rapport $N \times 6,38$ du lait/présure en poudre qui variait de $300: 1$ à $30: 1$.

Correlations between : i) Times at inflexion points $\left(t_{i r}\right)$ of refractometric curves and gelification times $\left(t_{g v}\right)$ calculated from viscosimetric curves $(--)$; ii) Between $\left(t_{i r}\right)$ and gelification times $\left(t_{g r}\right)$ calculated from the refractometric curves (...) and between $\left(t_{g r}\right)$ and $\left(t_{g v}\right)(-)$. Experimental conditions were the same as described in figure 2 except the $N \times 6.38$ milk/rennet ratio was varying between $300: 1$ and $30: 1$.

La comparaison des signaux obtenus à partir des deux capteurs sur des échantillons de lait amenés à des $\mathrm{pH}$ compris entre 5,6 et 6,6 montrait également l'identité du phénomène détecté. En effet, les évolutions des temps de gélification calculés à partir des courbes viscosimétriques et réfractométriques en fonction du $\mathrm{pH}$ du lait emprésuré sont décrits par des équations très similaires (fig. 7) :

$$
\begin{array}{ll}
\mathrm{t}_{\mathrm{gv}}=\frac{\mathrm{pH}}{0,203-0,030 \mathrm{pH}} & - \text { avec } \mathrm{r}=0,998 \\
\mathrm{t}_{\mathrm{gr}}=\frac{\mathrm{pH}}{0,211-0,032 \mathrm{pH}} & - \text { avec } \mathrm{r}=0,999
\end{array}
$$

L'analyse des courbes $\mathrm{V}=\mathrm{f}(\mathrm{t})$ dans l'échelle de $\mathrm{pH}$ étudiée montrait que la viscosité initiale du lait est légèrement affectée par la baisse du $\mathrm{pH}$. La décroissance était de $0,23 \mathrm{mPa}$.s par unité $\mathrm{pH}$ (fig. 8). Par contre, dans les conditions 


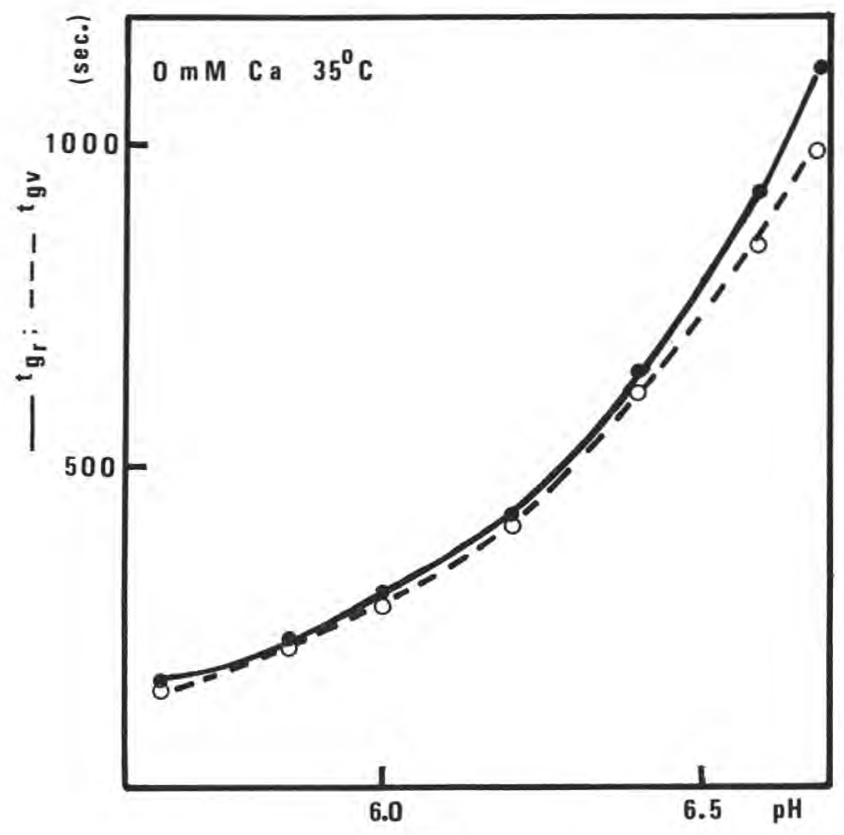

Fig. 7

Effet du $p H$ sur les temps de gélification calculés à partir des courbes réfractométriques (courbes en trait plein) et à partir des courbes viscosimétriques (courbes en pointillé). Les conditions expérimentales étaient les mêmes que celles de la figure 3 mais le pH était abaissé par addition d' $\mathrm{HCl} 0,5 \mathrm{~N}, 1 \mathrm{~h}$ avant l'addition de présure.

Effect of $p H$ on gelification times calculated from refractometric curves (continuous line) and from viscosimetric curves (dotted line). Experimental conditions were the same as described in figure 3 except the milk $\mathrm{pH}$ was lowered by adding $0.5 \mathrm{HCl}, 1$ hour before rennet addition.

expérimentales suivies, les viscosités aux points d'inflexion et aux maxima des courbes $\mathrm{V}=\mathrm{f}(\mathrm{t})$ étaient quasiment indépendantes du $\mathrm{pH}$.

Dans la zone de $\mathrm{pH}$ étudiée, la valeur du signal initial de sortie du réfractomètre ne semblait pas affectée. Les variations observées étaient inférieures ou du même ordre de grandeur que celles inhérentes au fonctionnement du capteur ou à la préparation des échantillons. Néanmoins, pour appréhender l'influence éventuelle du $\mathrm{pH}$ d'emprésurage sur les signaux du capteur réfractométrique, les relations entre cette valeur technologique et les rapports des valeurs initiales de ces signaux aux valeurs aux points d'inflexion (maxima des courbes $\mathrm{dR} / \mathrm{dt}$ ) et aux valeurs maximales des courbes $R=f(t)$ étaient déterminées (fig. 9) et comparées aux mêmes rapports calculés à partir des courbes viscosimétriques (fig. 8). Le rapport $R_{\text {inf }} / R_{\text {init }}$ était très peu dépendant du $\mathrm{pH}$ d'emprésurage; il variait de 1,05 à 1,02 entre $\mathrm{pH} 6,7$ et $\mathrm{pH} 6,4$ puis restait constant. Le rapport $\mathrm{R}_{\max } / \mathrm{R}_{\text {init }}$ semblait plus affecté par le pH. A pH 6,6, il était de 1,25. Entre pH 6,4 et pH 6,0 il passait par un minimum égal à 1,18 pour remonter légèrement ensuite jusqu'à 1,21 à $\mathrm{pH} 5,6$. A cette dernière valeur de $\mathrm{pH}$, il était observé au temps correspondant au maximum de la courbe $\mathrm{R}=\mathrm{f}(\mathrm{t})$ un début d'exsudation de lactosérum sur le gel adhérant à la fenêtre prismatique du réfractomètre. 


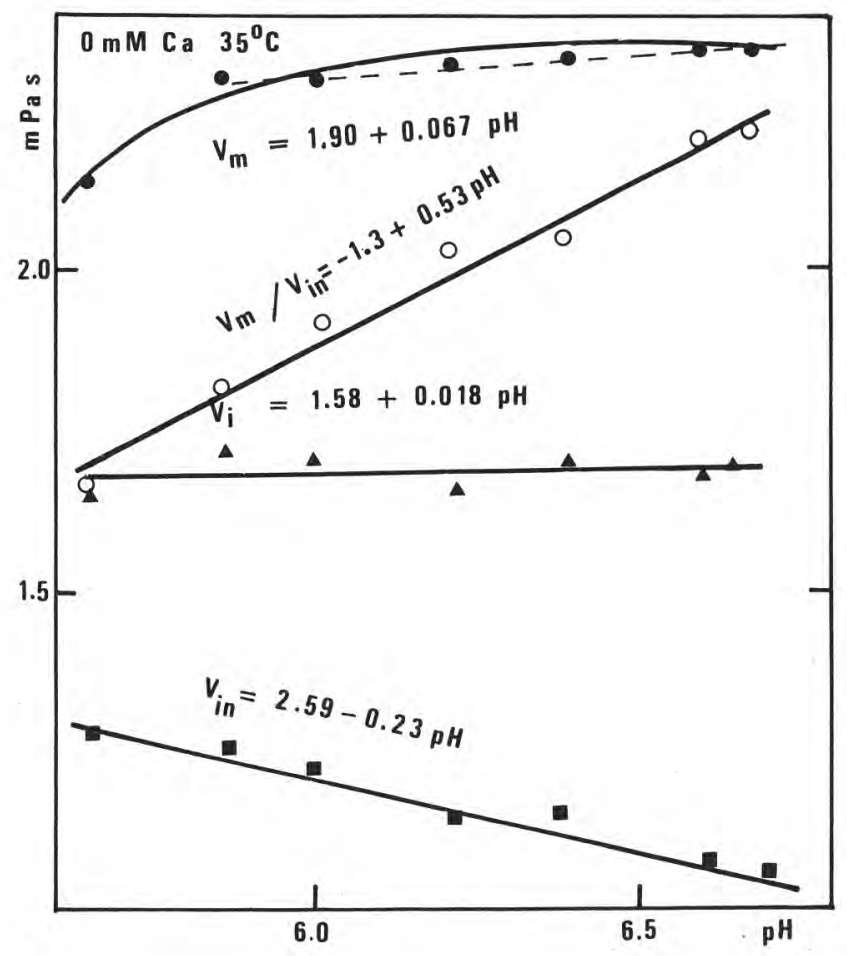

Fig. 8

Effet du pH sur la viscosité apparente du lait au cours de sa coagulation par la présure. Les conditions expérimentales étaient les mêmes que celles de la figure 7.

Effect of $p H$ on apparent viscosity of rennet gelifying milk. Experimental conditions were the same as described in figure 7.

- : in : Valeur initiale de la viscosité apparente.

Initial value of apparent viscosity.

A : i : Valeurs de la viscosité apparente aux maxima des courbes $d v / d t$. Apparent viscosity values at maxima of curves $d v / d$.

- : m : Valeurs des viscosités maximales.

Maxima values of apparent viscosities.

$0: \mathrm{m} /$ in $_{\text {: }}$ Rapport entre la viscosité maximale et la viscosité initiale.

Maximal to initial viscosity ratio.

\section{Discussion}

Les excellentes corrélations, mises en évidence au cours de cette étude, entre les signaux de sortie du capteur réfractométrique et les mesures de viscosité démontrent solidement que les deux capteurs mesurent le même phénomène, à savoir le changement d'état physique du lait sous l'effet de la présure et l'évolution du gel formé. Aussi, sous réserves de multiples confirmations quant à l'effet des différents facteurs intervenant dans les caractéristiques rhéologiques en site 


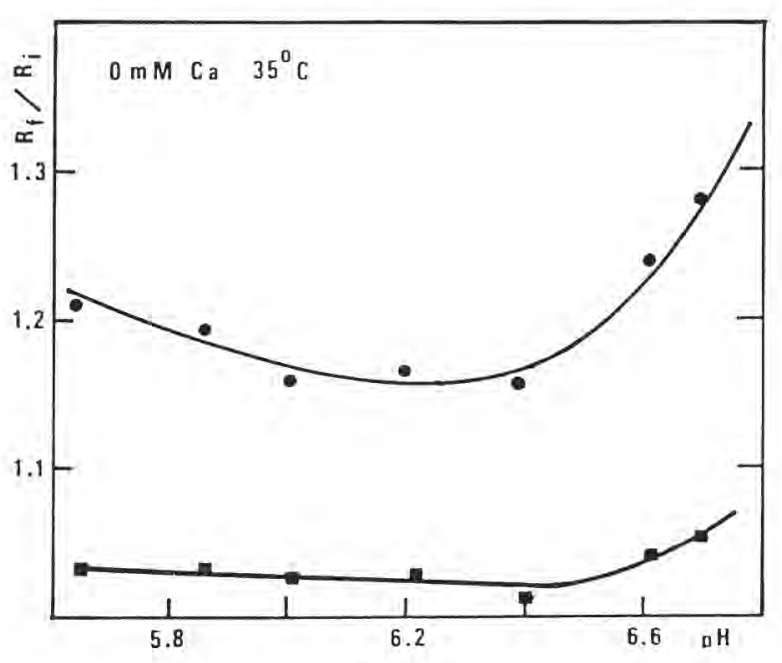

Fig. 9

Effet du pH sur les signaux de sortie relatifs du réfractomètre. Les conditions expérimentales étaient les mêrnes que celles de la figure 7 .

Effect of $\mathrm{pH}$ on output relative refractometric signals. Experimental conditions were the same as described figure 7.

- Rapport $R$ aux points d'inflexion / $R$ initial du signal réfractométrique. Inflexion point / initial $R$ levels of output refractometric signals.

○ : Rapport $R$ maxima / $R$ initial du signal réfractométrique.

Maximal $R /$ initial $R$ levels of output refractometric signals.

industriel, le réfractomètre que nous avons testé pourrait être le capteur idéal pour la conduite automatique des cuves de fromagerie, car non seulement il permet de détecter le début de la coagulation et de suivre le durcissement du gel mais de plus, de par le principe même de sa mesure optique, il ne risque en aucune façon d'endommager le gel, au contraire de tous les rhéomètres à cylindre à mouvement rotatif ou sinusoïdal récemment proposés (GERVAIs et Vermeire, 1983).

Deux causes principales peuvent être envisagées pour expliquer la variation du signal de sortie du réfractomètre au cours de la coagulation. La première est que les changements de conformation micellaire et la formation du réseau tridimensionnel du gel provoqueraient un accroissement de l'indice de réfraction du milieu, mais aucun phénomène semblable n'a été décrit dans la littérature, à notre connaissance. La seconde découle de la conception même du réfractomètre Recor utilisé (fig. 2).

En raison de la diffusion de la lumière par les micelles de caséine, il est certain que la zone sombre du prisme du réfractomètre n'est pas totalement noire comme cela est le cas avec les solutions transparentes de composés à bas poids moléculaires (sucres par exemple). Par ailleurs, la coagulation du lait modifie l'indice de diffusion de la lumière (DALGLEISH et al., 1981; HARDY et al., 1981) et c'est probablement ce phénomène qui influence le plus la variation du signal de sortie du réfractomètre. N'ayant pas actuellement la possibilité de mesurer séparément les signaux émanant des trois photodétecteurs, nous ne pouvons aller plus avant dans l'éventuelle confirmation de cette hypothèse. 
Le réfractomètre testé avait une résolution supérieure à $0,1 \%$ contre seulement $1 \%$ pour le viscosimètre. Il en résulte qu'il détectait beaucoup plus tôt la réorganisation micellaire conduisant à l'accroissement de viscosité du lait emprésuré. Toutefois, les temps de gélification calculés à partir de l'application de l'équation de SCOTT-Blair et BuRnett (1973) aux courbes $\mathrm{R}=\mathrm{f}(\mathrm{t})$ étaient systématiquement de $15 \%$ supérieurs à ceux calculés à partir des courbes $\mathrm{V}=\mathrm{f}(\mathrm{t})$. Aucune hypothèse ne nous apparaît plausible pour expliquer ce décalage.

$\mathrm{Si}$ le $\mathrm{pH}$ d'emprésurage influence similairement les valeurs des temps de gélification calculés à partir des courbes viscosimétriques et réfractométriques, il n'en est pas de même en ce qui concerne l'allure générale des deux familles de courbes. Mais la décroissance du rapport $\mathrm{V}_{\max } / \mathrm{V}_{\text {init }}$ en fonction du $\mathrm{pH}$ doit être relativisée en raison du principe même de la mesure viscosimétrique. En effet, l'adhérence du lait coagulé aux parois du cylindre en rotation décroît lorsque le $\mathrm{pH}$ baisse et la contrainte maximale mesurée n'est pas caractéristique de la rigidité réelle du gel puisque celui-ci est partiellement détaché de la paroi. Ce détachement du gel des surfaces en mouvement doit se produire d'autant plus rapidement que les gradients de vitesse imposés sont élevés ; or, dans nos expérimentations nous avons utilisé un gradient de vitesse de $2347 \mathrm{~s}^{-1}$, ce qui conduisait à une viscosité maximale de gel de $2,5 \mathrm{~m}$. Pas très éloignée de celle observée $\left(10^{4} \mathrm{mPas}\right)$ à des faibles gradients de vitesse $\left(0,1 \mathrm{~s}^{-1}\right)$ (KorolczuK et al., 1985).

L'accroissement du rapport $\mathrm{R}_{\max } / \mathrm{R}_{\text {init }}$ constaté en dessous de $\mathrm{pH}$ 6,0 est peut-être en relation avec le phénomène de synérèse constaté visuellement au niveau du gel adhérant à la fenêtre prismatique. Si cet accroissement du signal de sortie correspondait bien à une concentration du gel du fait de l'expulsion par le gel de lactosérum, le capteur réfractométrique pourrait non seulement être utilisé pour automatiser les opérations de découpe du caillé en fromagerie mais aussi celles de brassage, de chauffage et de soutirage.

Reçu le 15 mai 1986.

Accepté pour publication le 26 juin 1986.

\section{Références bibliographiques}

BOUdIER J.F., LUQUET F.M., VINCENT M., 1977. Capteurs de mesure quantitative. Etude expérimentale appliquée à l'industrie laitière : Turbidimétrie - Réfractométrie - Viscosimétrie. Ind. Alim. Agric., 945-958.

Culioli J., SHerman P., 1978. Rheological aspects of the renneting of milk concentrated by ultrafiltration. J. Texture Stud., 9, 257-281.

Dalgleish D.G., Brinkuis J., Payens T.A.J., 1981. The coagulation of differently sized casein micelles by rennet. Eur. J .Biochem, 119, 257-261.

Doumltard R., 1973. Rheological analysis of curd formation. J. Texture Stud., 4, 158-165.

Dumanski A., PichкovsкiI B.S., 1929. Kolloid Ztg 48-338 cité dans Casein and its industrial application by Sutermeister E. and Browne F.L., 1939. Reinhold Publ. New York U.S.A.

Gervais A., VeIRMEIRE D., 1983. A critical study and improvement of the cheese curd torsiometer. I .Texture Stud., 14, 31-45. 
GoULDEN J.D.S., 1963. Determination of S.N.F. in milk and unsweetened condensed milk from refractive index measurements. J. Dairy Res., 30, 411-418.

HaRdy J., Fanni J., Weber F., 1981. Etude de la coagulation du lait par photométrie de réflexion diffuse. Sci. Aliments, 1, 351-364.

Korolczuk J., Roignant M., MauboIs J.L., 1985. Computer data acquisition of sinusoidally varying stress and strain. J. Texture Stud., 16, 129-142.

Korolczuk J., Maubois J.L., Cardenas R., Grosclaude G., 1986. Computerized rheometric method for studying rennet coagulation of milk. Lait, 66, 99-115.

Mahaut M., 1981. Etude du contrôle de la teneur en matière sèche des produits obtenus lors de l'ultrafiltration du lait sur membrane. Techn. Lait., 956, 9-13.

Rice F.E., Miscall J., 1926. Sweetened condensed milk. IV. A refractometric method for determining total solids. J. Dairy Sc., 9, 140-152.

Robertson T.B., 1918. The Physical Chemistry of the proteins. Ed. Longmans, Green and Co, New York, p. 359-367.

Scott-Blair G., BuRnEtT J., 1963. An equation to describe the rate of setting of blood and milk. Biorheol., 1, 183-191.

STACEY K.A., 1961. The use of light-scattering for the measurement of the molecular weight and size of proteins. In: Analytical Methods of protein chemistry. Vol. 3, Ed. Alexander P. and Block R.J., Publ. Pergamon Press, New York. 